\title{
Challenges in sublingual airway ultrasound interpretation
}

\author{
Ban C. H. Tsui, MD · Carolyn M. W. Hui, MD
}

Received: 27 January 2009/Revised: 10 February 2009/Accepted: 13 February 2009/Published online: 4 March 2009

(c) Canadian Anesthesiologists' Society 2009

\section{To the Editor,}

In a recent Letter to the Editor published in the Journal, we described our initial experience of using a novel and simple technique for ultrasound (US) airway imaging by placing a small, high frequency, curved array scanner in the sublingual fossa. ${ }^{1}$ Through our ongoing research and reassessment, we have subsequently identified that our initial interpretation of the anatomical structures depicted in the US image of that article was inaccurate.

Using a sublingual imaging approach, we attempted to obtain a longitudinal view of the larynx by placing the probe sagittally and longitudinally under the patient's tongue (Fig. 1). Excellent probe-tissue contact provides stable images while avoiding contact with the soft palate, thereby circumventing an unpleasant gagging reflex. However, what we had failed to consider in our initial interpretation is that ultrasonographic imaging of the larynx and trachea is limited by the presence of an air-tissue interface which allows little or no transmission of the US signal. This US signal transmission barrier led to an illusion of the obtained images which was incorrect. Our initial observations were based on the expected anatomic relationship between the trachea, the esophagus and the epiglottis, which appeared to confirm with the spatial relationships shown on the US image.

Electronic supplementary material The online version of this article (doi:10.1007/s12630-009-9077-y) contains supplementary material, which is available to authorized users.

B. C. H. Tsui, MD $(\bowtie) \cdot$ C. M. W. Hui, MD

University of Alberta Hospital, Edmonton,

AB T6G 2G3, Canada

e-mail: btsui@ualberta.ca

URL: www.EdmARA.ca
A study has been initiated to determine the feasibility of US imaging for airway assessment. We have observed that the presence of a poor US medium such as air, should appear as a hyperechoic artifact, which, in turn, can obscure US visualization of any structure posterior to that artifact. Specifically, the epiglottis is suspended in air, and the esophagus is posterior to the air-filled trachea. Thus, it should not able to obtain clear US images of these structures with the US beam orientated from antero-posteriorally, and cephalad to caudad, at the level of the sublingual fossa. To confirm our suspicion, we performed the sublingual US on ourselves. It was found that with the probe in situ, pushing up the underside of the chin resulted in corresponding compression of the dark hypoechoic structure on the US screen (Fig. 2). This excludes the possibility that this structure is the airway. We also found that we could move the hyperechoic structure on the US image by external manipulation of the hyoid. Therefore, it is now apparent that the dark anechoic structure (Fig. 1) which was originally interpreted as the trachea, is actually the geniohypoid muscle. The hyperechoic structure originally thought to have been the epiglottis, is the hypoid bone. During swallowing (see Electronic supplementary material), a dynamic view of elevation of this distinct hyperechoic structure depicts the hyoid bone being pulled anteriorly by the geniohyoid muscle.

Anesthesiologists may encounter considerable difficulty during identification of target structures when learning US techniques. This may lead to frustration and failure, deterrents in themselves from adopting this technology. So what lessons have we learned from this experience? We have been reminded, once again, that US visualization is indirect, and that images are subject to individual interpretation depending on the equipment being used, and individual experience and training. Instead of attempting to 
Fig. 1 Schematic diagram and ultrasound image using sublingual scanning approach
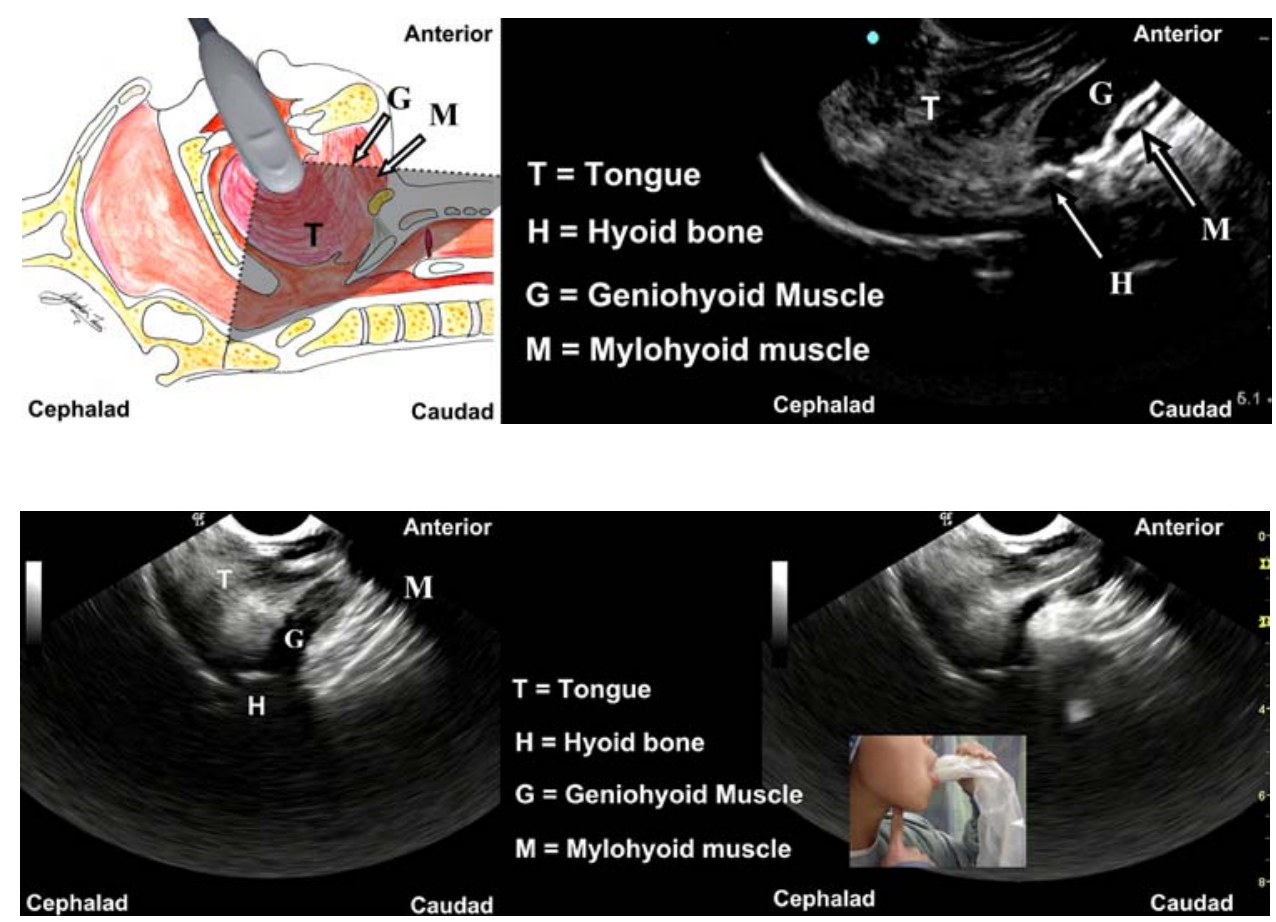

Fig. 2 Sublingual ultrasound (Left) Before and (right) after pushing up the underside of the chin resulting in corresponding compression of the dark hypoechoic structure on the ultrasound screen

\section{Reference}

1. Tsui BC, Hui CM. Sublingual airway ultrasound imaging (Letter). Can J Anesth 2008; 55: 790-1.

\begin{abstract}
US beam.
\end{abstract}
identify the target structure as the initial step in US imaging, one should first review the relevant anatomy of the region being imaged, and consider the physics of the Conflicts of interest None declared. 